\title{
Teaching the Equivalence Principle through a Combination of Real-Life Experiments and Computer Simulations ${ }^{\dagger}$
}

\author{
Efstratios Kapotis ${ }^{1, *(\mathbb{D})}$ and Panagiotis Tsakonas ${ }^{2}$ \\ 1 Department of Phyiscs, National and Kapodistrian University of Athens, 15784 Athens, Greece \\ 2 Department of Informatics, University of Pireaus, 18534 Pireas, Greece; ptsakon@unipi.gr \\ * Correspondence: ekapotis@phys.uoa.gr; Tel.: +30-697-687-3683 \\ + Presented at the 1st Electronic Conference on Universe, 22-28 February 2021; Available online: \\ https://ecu2021.sciforum.net/.
}

Citation: Kapotis, E.; Tsakonas, P. Teaching the Equivalence Principle through a Combination of Real-Life Experiments and Computer Simulations . Phys. Sci. Forum 2021, 2, 6. https://doi.org/10.3390/ ECU2021-09281

Academic Editor: Magdalena Kersting

Published: 22 February 2021

Publisher's Note: MDPI stays neutral with regard to jurisdictional claims in published maps and institutional affiliations.

Copyright: (c) 2021 by the authors. Licensee MDPI, Basel, Switzerland. This article is an open access article distributed under the terms and conditions of the Creative Commons Attribution (CC BY) license (https:// creativecommons.org/licenses/by/ $4.0 /)$.

\begin{abstract}
The need to transform scientific knowledge to materials suitable for teaching school students is a constant challenge for the educational community. Although it has been more than a century since quantum mechanics and the theory of relativity were established, both topics continue to be treated as modern physics, and only recently did they begin to be taught to students of levels prior to higher education. The work at hand is part of a larger effort to introduce the general theory of relativity in schools. To this end, we have devised appropriate experiments and computer simulation software. In particular, we present an educational simulation software that we created for the teaching of the Equivalence Principle. The implementation was applied to 120 undergraduate students of the Pedagogical Department of the University of Athens who do not major in physics but will be expected to teach young students the basic principles of relativity. The simulation software enables the user to measure forces inside a gravitational field and compare them to those exerted on bodies being accelerated. The controls incorporated in the software aim to motivate students to perform a variety of experiments, investigating every possible combination of parameters, in the hope to help them overcome most of the learning difficulties highlighted by previous research. The encouraging results of the research confirm the need to continue to fill gaps in the fragmented instruction of physics in schools.
\end{abstract}

Keywords: general relativity; equivalence principle; simulation software; virtual experiments

\section{Introduction}

\subsection{Necessity}

The Equivalence Principle (EP or Principle) was of major importance in the formulation of the General Theory of Relativity (GR) by Albert Einstein. Its role is equally significant in teaching the subject. The inability to distinguish between an accelerated system and a homogeneous gravitational field was considered by Einstein himself as the happiest thought of his life [1]. Every textbook dealing with GR references the Principle [2]. In their vast majority, authors present the Principle in the introductory chapter in order to emphasize that a free fall is equivalent to inertial motion. It is also used as a means of interpreting the bending of light, time dilation in gravitational fields and gravitationa redshift.

\subsection{Literature Review}

Researchers trying to promote the semantic link between Special and General Relativity [3] or introduce the General Theory in Secondary and University Education suggest that the EP, its consequences and the thought experiment of the elevator (with an adequately simple mathematical formalism) suffice to familiarize students with the scientific and cultural value of the GR [4]. Furthermore, they state that, in order to derive results from the 
Principle (light aberration and gravitational time dilation), one must introduce the notion of thought experiments [5]. In recent years, research on the didactic transformation of the General Theory and its incorporation in all levels of education has led to methods aiding the teaching of the Principle, either as a thought experiment described in class, or as a free fall experiment performed in the classroom or in special surroundings. The next step is to make the connection with other "great ideas" of relativity. To this end, experimentation and educational software are frequently combined [6]. While, in attempting to teach the General Theory, one concludes that the Principle is suitable for motivating students [7], unfortunately it is not free of comprehension difficulties. Students face difficulties in applying the Principle [8], especially in areas where a gravity field is present [9], because they tend to use their everyday experience or the idea that a body inside a room located outside a gravitational field hovers independently of the room's movement [10].

\section{Methods}

This work is part of larger research conducted internationally by a major part of the research community that aims to make Einstein's physics accessible to undergraduate students who do not major in Physics and to school students of all education stages. Here, we present and evaluate the combination of traditional experimentation in the classroom with educational simulations used to teach the EP. At an earlier time, we constructed an improvised experimental device used by students to experiment in the classroom [11]. Through the educational evaluation of this device, we found out that it has a positive impact on learning results, but students face a number of limitations during the experimentation process. To the end of lifting these limitations, we developed appropriate simulation software. The combination of real-life experiments and computer-aided simulations was integrated in a teaching process focusing on the evaluation of achieved results.

\subsection{Research Question}

Does the use of original demonstrative experimentation using simple means paired with interactive computer simulations, incorporating control of variables that, according to research, pose difficulties to students in reference to the EP, promote positive learning outcomes of a higher level compared to those accomplished by the usual ways of teaching?

\subsection{Objectives}

Our educational proposal and intervention aim to:

- Create digital simulations that complement traditional experimentation, in order to facilitate students' understanding, while being suitable for use in every modern device;

- Compose an educational sequence-a worksheet that utilizes digital teaching tools (video, images, sounds, text open for concurrent processing, hyperlinks, digital evaluation tools);

- Conduct and evaluate a teaching intervention based on the proposed experimentation.

\subsection{Creating Experiments}

Using a simple cardboard box, we created an original improvised elevator (Figure 1) that can perform free fall or move at constant acceleration with the aid of a simple system of pulleys. We used it to demonstrate to students many results concerning the EP. A camera, recording the interior of the box, shows the various readings on a spring scale or the change of the flow of a water current emerging from the side surface of a vessel. A number of devices proposed by students performing the educational experimentation may be fitted inside the spacious box. 


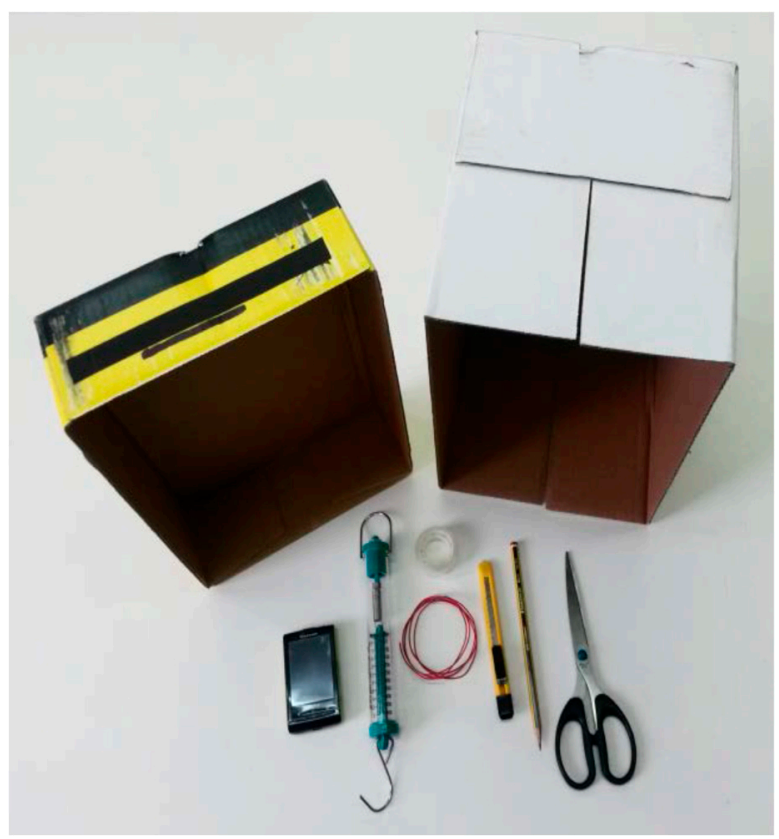

(a)

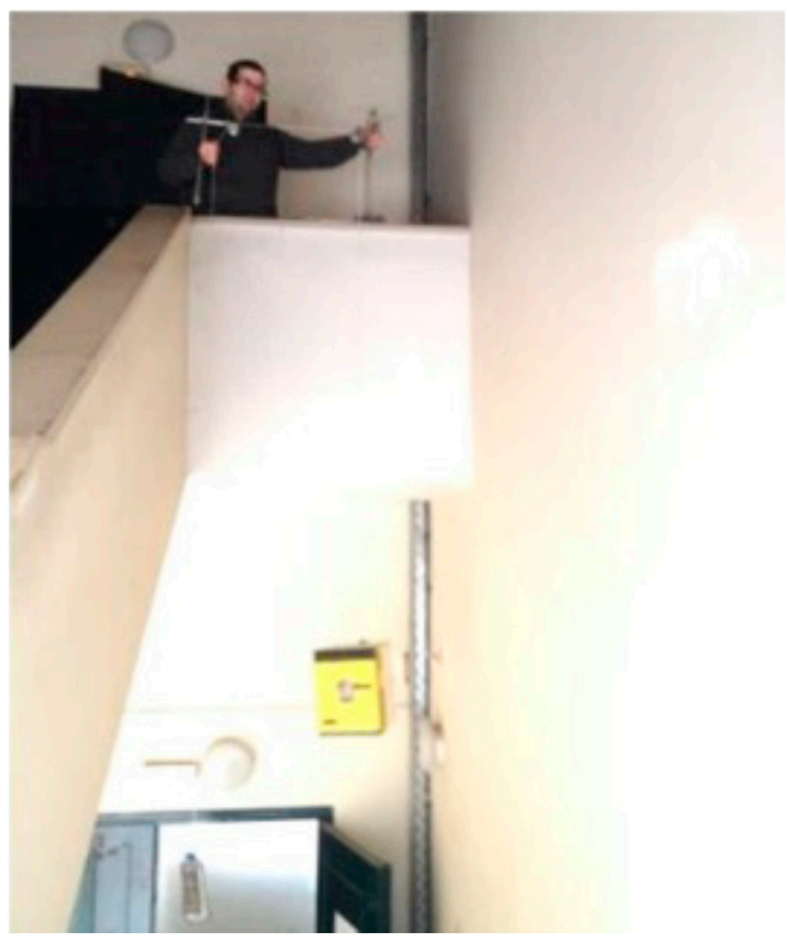

(c)

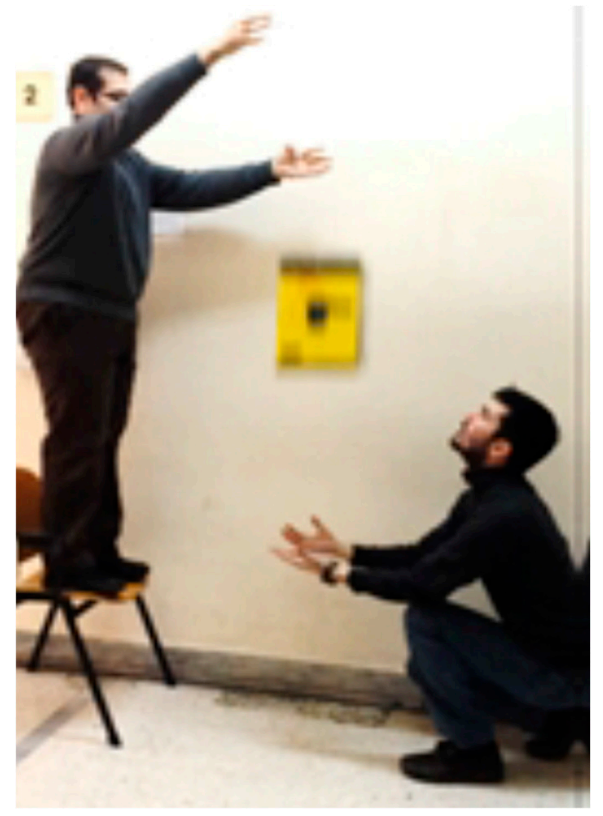

(b)

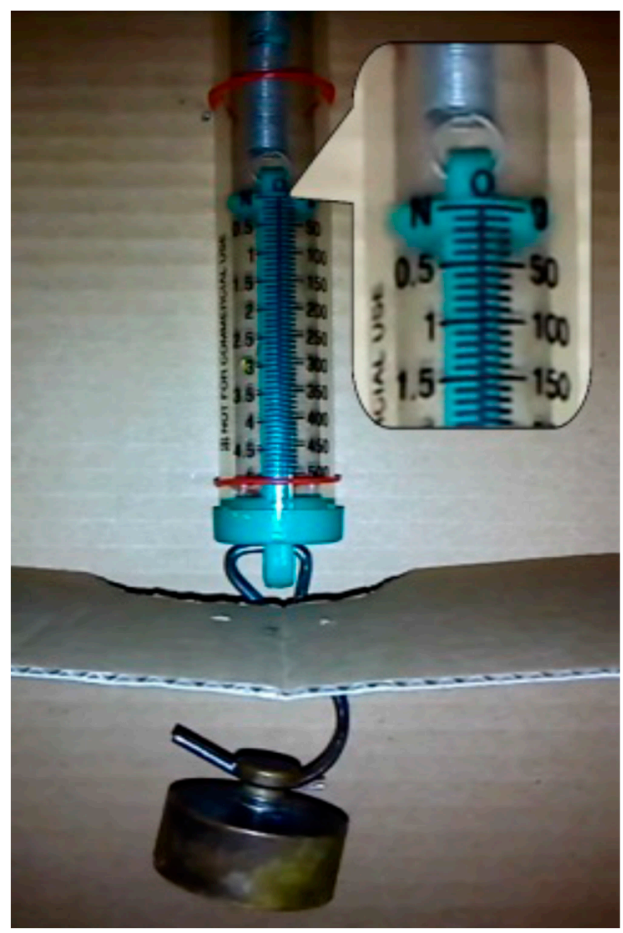

(d)

Figure 1. Improvised elevator for conducting experiments concerning the EP: (a) materials needed to assemble the device; (b) elevator in free fall; (c) elevator moving upwards at constant acceleration; (d) spring scale measurement during free fall equaling $0 \mathrm{~N}, 0 \mathrm{~g}$, while a $100 \mathrm{~g}$ body is suspended from the spring's free endpoint.

A limitation of such a device is the inability to perform measurements for various directions of movement and/or outside gravitational fields. To overcome this particular obstacle, we created appropriate simulation software (Figure 2), enabling the user to experiment in various situations by controlling a number of variables (elevator moving at constant velocity or constant acceleration, direction of movement) (http:/ / users.sch.gr / ptsakon/RG_elevator_EN/RG_elevator.html, last accessed 30 June 2021). By changing the 
values of these parameters and reviewing the results, students manage to overcome the difficulties recorded in previous research [9].
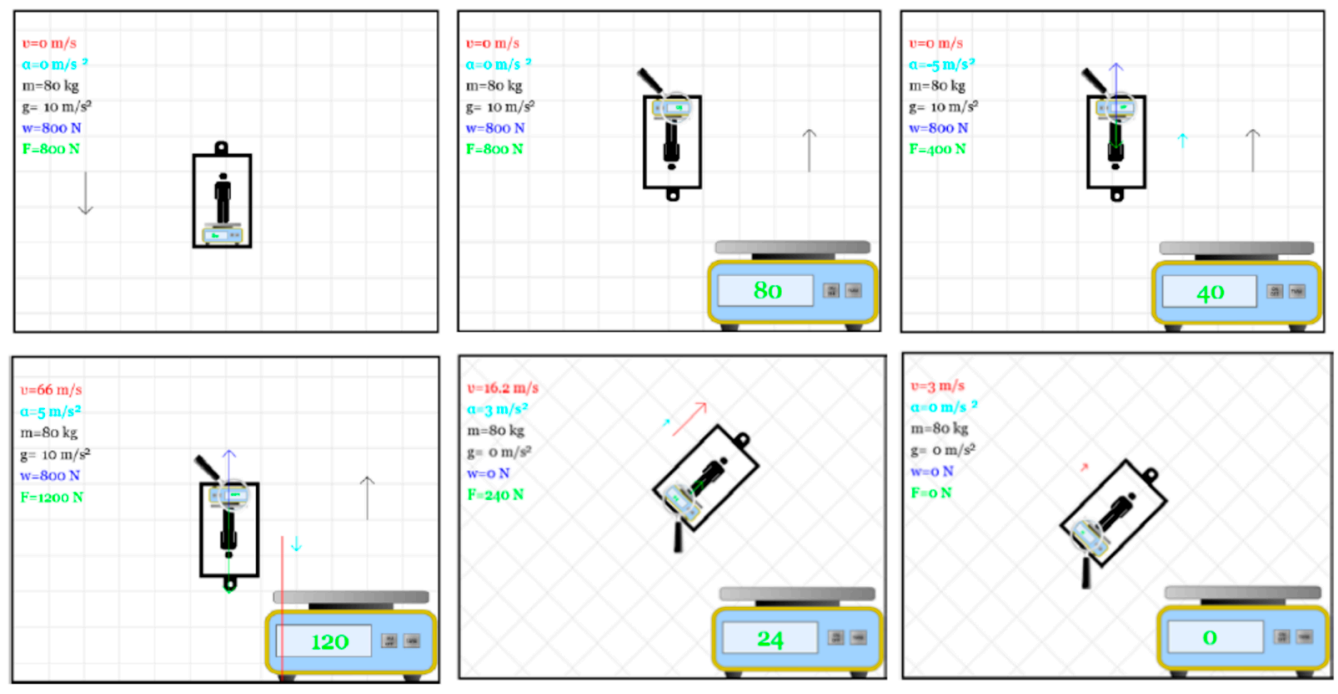

Figure 2. Indicative software snapshots showing a number of ways of elevator movement (various values of velocity and acceleration, as well as direction of movement), while measuring magnitudes of mass, weight, normal force and showing corresponding vectors.

\subsection{Digital Environment and Worksheet}

The worksheet was created in the form of a typical interactive web page, embedding experimentation, simulations (Figure 3), images and videos used to trigger students' interest, as well as interactive co-authoring documents used to record assumptions, measurements, conclusions and generalizations. The educational stages of the worksheet are the ones proposed in the Scientific/Educational Method by Inquiry [12].

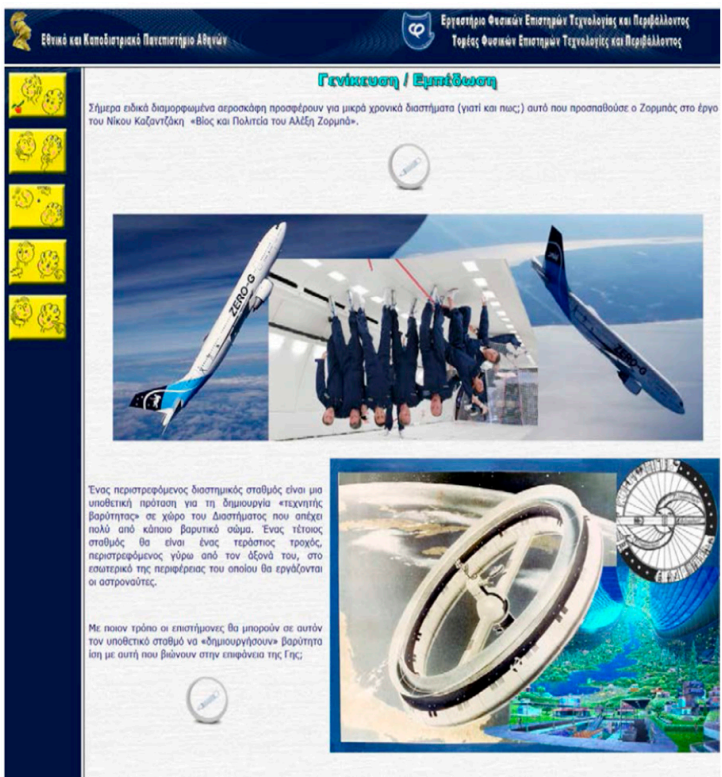

(a)

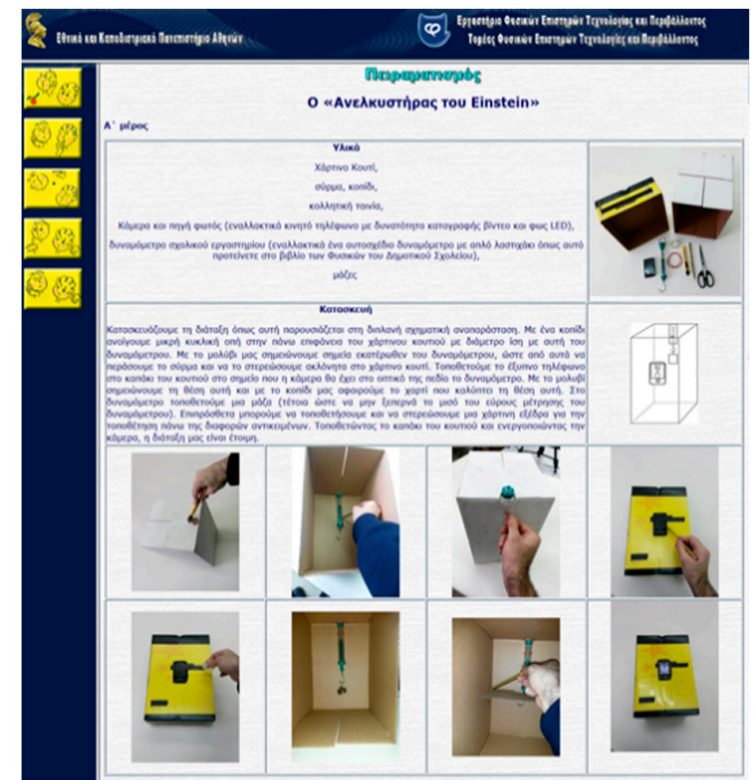

(b)

Figure 3. Sample steps of (a) Experimentation and Generalization-Consolidation of knowledge from the digital worksheet showing weightlessness in free fall flight, designs for future space stations recreating gravity through acceleration and (b) instructions on constructing an improvised "Einstein elevator-box". The rest of the steps are accessible via the left side buttons. 


\subsection{Research Sample and Evaluation Tool}

The research sample consisted of 120 (19 male and 101 female) undergraduate students of the Department of Primary Education of the University of Athens, students de facto not majoring in Physics. The sample was selected at random from the students available at the time the research was carried out.

In order to record the knowledge of the students along with their level of comprehension, we composed a questionnaire consisting of five multiple choice questions, each one presenting a set of three options. Students had to pick one of the suggested answers, which were selected on the basis of addressing frequent misconceptions and difficulties. In order to answer correctly, students were expected to have adequately comprehended and be able to correctly apply the EP and its consequences in complex situations. Three of these questions are reproduced in Figure 4.

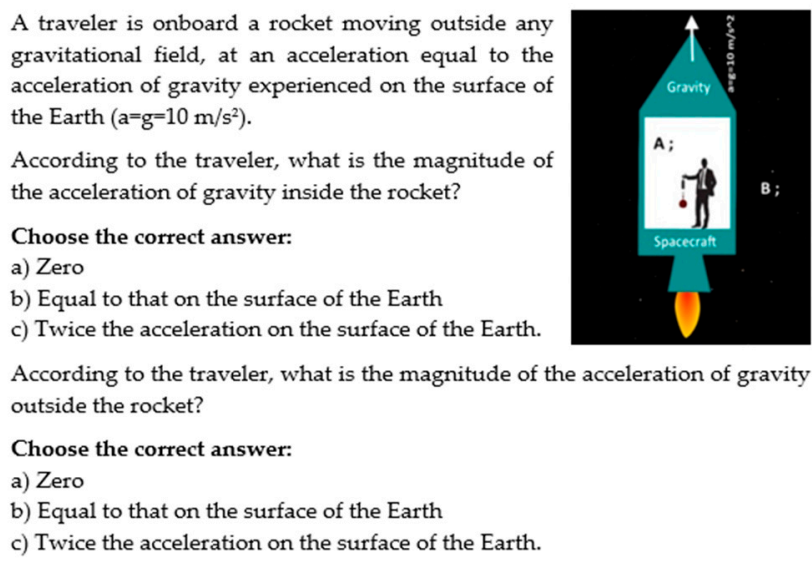

(a)

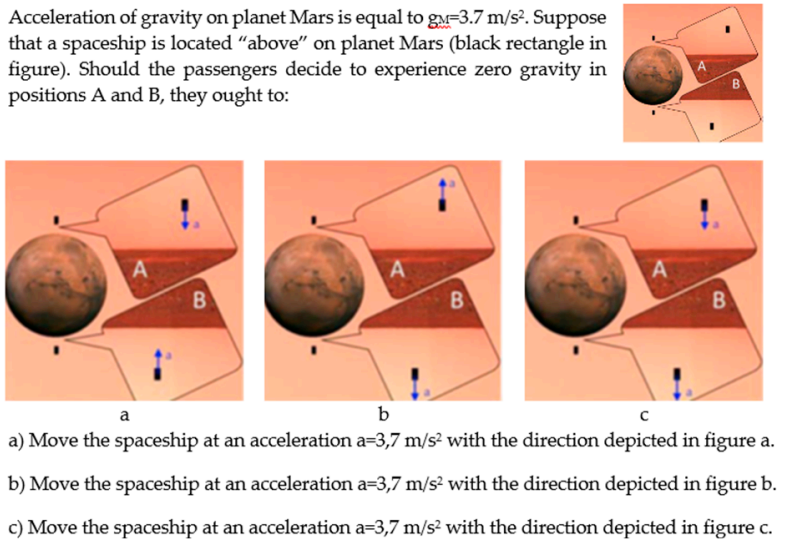

(b)

Figure 4. Three of the multiple-choice questions from the evaluation questionnaire of the intervention: (a) Questions 3 and 4 , (b) Question 5.

\subsection{Research Process}

In order to check the validity of the educational and evaluation material of our approach, a group of specialists, consisting of two physics majors with a postgraduate title on teaching science and a PhD specialized on the General Theory, were called upon to offer their expertise. Afterwards, we conducted formative pilot research with 10 students of the Department. After this step, the educational material and the questionnaire were given their definitive form.

To better serve our research requirements, two different sets of students were composed, each one consisting of 60 individuals. The first served as the control group, where the traditional method of teaching was applied, while the other was the test group-the members of which formed 20 smaller teams of three students each. Both groups were handed questionnaires before (pre-tests) and after (post-test) our intervention.

\section{Results and Discussion}

We used the $x^{2}$ test in order to perform the statistical analysis of the collected data and reach conclusions, since our research variables are measured on a tactical scale and include two nominal independent groups.

The statistical processing of the results showed that the two groups were equivalent on their knowledge of the EP and its consequences, since no statistically significant difference was observed in any question of the pre-tests. After the teaching intervention, we marked a statistically significant difference in the results of the post-test for the majority of the questions with the test group improving the level of comprehension of the EP, as shown in Table 1. 
Table 1. Pre- and post-test comparative results by question number.

\begin{tabular}{ccc}
\hline Question \# & Pre-Test $\left(\mathbf{x}^{\mathbf{2}}\right.$ Test) & Post-Test $\mathbf{x}^{\mathbf{2}}$ Test) \\
\hline $\mathbf{1}$ & Pearson Chi-Square $0.409, p=0.522$ & Pearson Chi-Square 7.566, $p=0.006$ \\
\hline $\mathbf{2}$ & Pearson Chi-Square $0.240, p=0.624$ & Pearson Chi-Square 24.422, $p=0.000$ \\
\hline $\mathbf{3}$ & Pearson Chi-Square $0.136, p=0.713$ & Pearson Chi-Square 0.891, $p=0.345$ \\
\hline $\mathbf{4}$ & Pearson Chi-Square 0.657, $p=0.418$ & Pearson Chi-Square 0.874, $p=0.350$ \\
\hline $\mathbf{5}$ & Pearson Chi-Square 0.376, $p=0.540$ & Pearson Chi-Square 32.475, $p=0.000$ \\
\hline
\end{tabular}

Although in absolute numbers the test group appears to prevail, in two of the questions no statistically significant difference was observed. We included these questions ( 3 and 4 ) intending to address and inspect the findings of other research studies that state "students limit the area of the gravitational field inside the lab" [8], although the size of their sample and their general approach allow for preliminary results and approximations, rather than generalizations. At this stage, but also in previous research concerning students' difficulties, we were unable to validate this report $[9,11]$.

\section{Conclusions}

Taking into account (a) the course of implementation and educational use of an original real-life experimentation with simple materials; (b) the development of simulation software that, besides being scientifically accurate, is structured in order to contribute to the elimination of difficulties students face; (c) the synthesis of an integrated way for the educational approach of the EP; and (d) the comparative assessment of our proposal in view of similar work suggested in the literature and used by teachers, we believe that our work answers the research question proposed.

We nevertheless need to stress that, in both educational and scientific research, a generalization of the findings is safe only to the extent that results are based on scientific methodology requiring repeatability and application from independent researchers. In this view, we believe that the proposal and its application must be tested on a large scale, evaluated and published. Such actions are necessary to ensure the successful adaptation of the scientific model of the General Theory of Relativity to an educational model, incorporating real and virtual experiments along with digital technologies, bridging a gap ascertained internationally. Furthermore, we need extensive research of learning outcomes and an improvement of conceptual understanding, ensuring that students comprehend the principles of relativity in depth rather than experiencing an illusion of understanding. To this end, we agree that conducting interviews is of vital importance [7].

In conclusion, we deem it necessary to mention some easy to implement suggestions that will aid students to master the EP and, in general, the Theory of Relativity. Parts of the curriculum may serve a dual purpose, i.e., function as pre-existing knowledge and as a useful working process that leads seamlessly to the introduction of Relativity, make the material taught less fragmentary and emphasize and highlight its unifying character. For example, when teaching Newton's laws in Classical Mechanics, one may describe the interesting yet "mysterious" equivalence between inertial and gravitational mass and refer to phenomena that would be radically different if such an equivalence did not hold. Additionally, in the study of Earth's gravity, one may examine the effects of rotation in the determination of weight and, from there, trigger a discussion about weightlessness in spaceships.

Supplementary Materials: The poster presentation is available online at https: / /www.mdpi.com/ article/10.3390/ECU2021-09281/s1.

\section{References}

1. Pais, A.B. Subtle is the Lord: The science and the life of Albert Einstein. Phys. Today 1983, 36, 81. [CrossRef] 
2. Di Casola, E.; Liberati, S.; Sonego, S. Nonequivalence of equivalence principles. Am. J. Phys. 2015, 83, 39-46. [CrossRef]

3. Drake, S.P. The equivalence principle as a stepping stone from special to general relativity: A Socratic dialog. Am. J. Phys. 2006 74, 22-25. [CrossRef]

4. Levrini, O. Reconstructing the Basic Concepts of General Relativity from an Educational and Cultural Point of View. Sci. Educ. 2002, 11, 263-278. [CrossRef]

5. Zahn, C.; Kraus, U. Sector models-A toolkit for teaching general relativity: I. Curved spaces and spacetimes. Eur. J. Phys. 2014, 35, 39-46. [CrossRef]

6. Kersting, M.; Toellner, R.; Blair, D.; Burman, R. Gravity and warped time-clarifying conceptual confusions in general relativity. Eur. J. Phys. 2014, 35, 39-46. [CrossRef]

7. Pössel, M. Relatively complicated? Using models to teach general relativity at different levels. arXiv 2018, arXiv:1812.11589.

8. Bandyopadhyay, A.; Kumar, A. Probing students' understanding of some conceptual themes in general relativity. Phys. Rev. Spec. Top. Phys. Educ. Res. 2010, 6, 020104. [CrossRef]

9. Gousopoulos, D.; Kapotis, E.; Kalkanis, G. Students' difficulties in understanding the basic principles of Relativity after standard instruction. In Proceedings of the ESERA Conference, Helsinki, Finland, 31 August-4 September 2015; Lavonen, J., Juuti, K., Lampiselkä, J., Uitto, A., Hahl, K., Eds.; ESERA: Utrecht, The Netherlands, 2005.

10. Velentzas, A.; Halkia, K. The Use of Thought Experiments in Teaching Physics to Upper Secondary-Level Students: Two examples from the theory of relativity. Int. J. Sci. Educ. 2013, 35, 3026-3049. [CrossRef]

11. Kapotis, E.; Kalkanis, G. Einstein's Elevator in Class: A Self-Construction by Students for the Study of the Equivalence Principle. Phys. Teach. 2016, 54, 404-407. [CrossRef]

12. Micro-Kosmos. Available online: http://micro-kosmos.uoa.gr/en/ekp_protaseis/methodology.pdf (accessed on 30 January 2021). 